\title{
Deficiency of the microglial Hv1 proton channel attenuates neuronal pyroptosis and inhibits inflammatory reaction after spinal cord injury
}

Xuefei Li, Zhiyuan Yu, Weifeng Zong, Peng Chen, Jia Li, Minghuan Wang, Fengfei Ding, Minjie Xie, Wei Wang and Xiang Luo*

\begin{abstract}
Background: Spinal cord injury (SCl) causes neurological dysfunction with devastating consequences. SCI pathogenesis is accompanied by inflammasome activation and neuronal damage. But the spatial pattern and the time course of neuronal pyroptosis and apoptosis after $\mathrm{SCl}$ should be further elucidated. The microglial voltagegated proton channel (Hv1) is implicated in reactive oxygen species (ROS)-induced neuronal damage following ischemic stroke. However, there is a lack of quantification on the neuronal pyroptosis and apoptosis associated with microglial Hv1 after SCl.
\end{abstract}

Methods: We analyzed spatial and temporal characteristics of neuronal pyroptosis and apoptosis following SCl and investigated the effects of $\mathrm{Hv} 1$ deficiency on neuronal pyroptosis and the nod-like receptor 3 (NLRP3)

inflammasome pathway by using a mouse model of SCl. We tested the effects of Hv1-deficient microglia on ROS production in vivo and examined the relationship between ROS and neuronal pyroptosis in vitro.

Results: We observed that apoptosis was detected closer to the injury core than pyroptosis. The incidence of neuronal apoptosis peaked on day 1 after $\mathrm{SCl}$ and occurred before pyroptosis. Hv1 deficiency reduced neuronal apoptosis and NLRP3-inflammasome-mediated pyroptosis, improved axonal regeneration, and reduced motor deficits. SCI led to elevated ROS levels, whereas Hv1 deficiency downregulated microglial ROS generation. In vitro, ROS upregulated neuronal pyroptosis and activated the NLRP3 inflammasome pathway, both of which were reversed by addition of a ROS scavenger. Our results suggested that microglial Hv1 regulated neuronal apoptosis and NLRP3-induced neuronal pyroptosis after SCl by mediating ROS production.

Conclusion: Following $\mathrm{SCl}$, neuronal pyroptosis lasted longer and occurred farther away from the injury core compared with that of neuronal apoptosis. Microglial Hv1 deficiency downregulated microglial ROS generation and reduced apoptosis and NLRP3-induced neuronal pyroptosis. Our findings may provide novel insights into Hv1associated mechanisms underlying neuronal damage after SCl.

Keywords: Spinal cord injury, Microglia, Voltage-gated proton channel, Pyroptosis, NLRP3 Inflammasome, Reactive oxygen species, Apoptosis

\footnotetext{
* Correspondence: flydottjh@163.com

Department of Neurology, Tongji Hospital, Tongji Medical College, Huazhong

University of Science and Technology, Wuhan 430030, China
}

(c) The Author(s). 2020 Open Access This article is licensed under a Creative Commons Attribution 4.0 International License, which permits use, sharing, adaptation, distribution and reproduction in any medium or format, as long as you give appropriate credit to the original author(s) and the source, provide a link to the Creative Commons licence, and indicate if changes were made. The images or other third party material in this article are included in the article's Creative Commons licence, unless indicated otherwise in a credit line to the material. If material is not included in the article's Creative Commons licence and your intended use is not permitted by statutory regulation or exceeds the permitted use, you will need to obtain permission directly from the copyright holder. To view a copy of this licence, visit http://creativecommons.org/licenses/by/4.0/ The Creative Commons Public Domain Dedication waiver (http://creativecommons.org/publicdomain/zero/1.0/) applies to the data made available in this article, unless otherwise stated in a credit line to the data. 


\section{Introduction}

Traumatic spinal cord injury (SCI) results in disability with frequently devastating consequences that affect long-term health and employment status [1]. SCI pathophysiology is initiated by mechanical tissue damage disrupting anatomical and functional integrity of the spinal cord, which contributes to secondary detrimental events. Neuroinflammation plays an important role in secondary neuronal damage following SCI [2]. The inflammatory reaction begins at the lesion site and then expands to surrounding tissue during the secondary phase [3]. A persistent inflammatory microenvironment and the presence of reactive oxygen species (ROS) are potential factors that impede injury repair following SCI $[4,5]$.

Microglia are major contributors to the inflammatory response after central nervous system (CNS) injury [6]. The voltage-gated proton channel (Hv1) maintains physiological intracellular $\mathrm{pH}$ and is abundantly expressed on the surface of microglia [7]. Although Hv1 is specifically expressed in microglia in the nervous system, Hv1 can influence neurons by regulating microglial functions under pathological conductions. Previous studies found that Hv1 was implicated in pathological NADP $\mathrm{H}$-oxidase-mediated ROS generation leading to neuronal apoptosis in ischemic stroke $[8,9]$. Our previous study has shown that ROS levels and pro-inflammatory cytokines are reduced in Hv1-deficient $\left(\mathrm{Hv}^{-/-}\right)$microglia compared to those in wild-type (WT) microglia after oxygen-glucose deprivation in vitro [10]. However, the contribution of microglial Hv1 in neuronal damage and the underlying molecular mechanisms following SCI remain unknown.

Neuronal death via apoptosis, pyroptosis, or necroptosis is regulated through activities of various host proteins that induce different biological outcomes [11, 12]. In contrast to apoptosis, neuronal pyroptosis is a form of cellular death induced by inflammatory caspases (e.g., caspase-1, caspase- 4 and caspase-5, or caspase-11) implicated in ischemic stroke and SCI [13, 14]. However, little is known regarding the spatial and temporal distributions of neuronal pyroptosis and apoptosis following SCI. Cleavage and activation of the pore-forming effector protein, gasdermin D (GSDMD), has been shown to determine pyroptosis initiated by inflammasomes [15]. The nod-like receptor 3 (NLRP3) inflammasome, the well-studied inflammasome, is a multiprotein complex consisting of nod-like receptor 3 (NLRP3), apoptosis-associated speck-like protein (ASC), and an effector pro-caspase-1 $[16,17]$. It is unknown whether microglial Hv1 can promote neuronal pyroptosis through the NLRP3 inflammasome after SCI. Here, we used a mouse model to analyze spatial and temporal patterns of neuronal apoptosis and pyroptosis following SCI. We examined whether Hv1 deficiency exerts neuroprotective effects through modulating inflammasome activity and neuronal pyroptosis. Our findings may contribute to the identification and development of a potential therapeutic strategy for individuals with SCI.

\section{Materials and methods Animals}

C57BL/6 female mice (wild-type, WT) were obtained from the Laboratory Animal Facilities of Hubei Center (Wuhan, China). $\mathrm{Hv1}^{-/-}$mice were kindly provided by Prof. Long-Jun Wu (Department of Cell Biology and Neuroscience, Rutgers University) and were generated as previous study [18]. The $\mathrm{Hv}^{-1-}$ genotype was confirmed using PCR, Western blotting, and immunofluorescent analysis of tail DNA, spinal cord protein, and tissue sections, respectively. Mice were maintained under a 12 h light/12-h dark cycle at $22{ }^{\circ} \mathrm{C}$ and were provided food and water ad libitum. All female mice were 10-12 weeks of age and weighed $20-25 \mathrm{~g}$ in weight. WT and $\mathrm{Hv1}^{-/-}$ mice were randomly distributed into sham-operation and SCI groups. All experiments and procedures were approved by the Institutional Animal Care and Use Committee of Tongji Medical College, Huazhong University of Science and Technology.

\section{Spinal cord injury model}

Prior to surgery, mice were anesthetized with $2 \%$ isoflurane. A T9-11 laminectomy was performed, and the spinal cord was exposed at T10. A 5-g weight was dropped from a height of $11 \mathrm{~mm}$ onto the exposed dorsal surface of the spinal cord using a modified NYC impactor (J\$K Seiko Electronic, China) [19]. Shamtreated mice underwent a laminectomy at T9-11 without spinal cord injury. The bladder was voided twice daily until the recovery of urinary function post-spinal cord injury (SCI). Locomotor function was evaluated using the Basso Mouse Scale (BMS) [20].

\section{Tissue preparation}

Animals were anesthetized and then perfused transcardially with $0.01 \mathrm{M}$ of phosphate-buffered saline (PBS) at $1,3,7,14$, and 28 days after treatment $(n=5$ for each time point). For Western blotting and ROS detection, spinal cord tissues-including the injury core and approximately $2 \mathrm{~cm}$ of surrounding tissue-were rapidly excised and frozen in cooled isopentane. For immunofluorescence, mice were perfused with $4 \%$ paraformaldehyde (PFA) in PBS and spinal cord tissues-including the injury core and approximately $2 \mathrm{~cm}$ of surrounding tissue-were dissected, fixed in PFA overnight at $4{ }^{\circ} \mathrm{C}$, and then transferred to $30 \%$ sucrose in $0.01 \mathrm{M}$ of PBS for 3 days. All the tissue samples were stored at $-80{ }^{\circ} \mathrm{C}$ until further use. Ten-micron sections were then 
prepared for Luxol fast blue (LFB) staining, immunofluorescence, and terminal dexynucleotidyltransferasemediated dUTP nick-end labeling (TUNEL) assays.

\section{Luxol fast blue staining}

Luxol fast blue (LFB) staining was used to assess lesion volumes after SCI. Briefly, spinal cord tissue sections at 14 and 28 days after treatment were incubated overnight in $0.1 \%$ LFB (Servicebio, Wuhan, China) at $60{ }^{\circ} \mathrm{C}$. Sample observations and image acquisitions were performed using a light microscope (BX51, Olympus, Japan).

\section{Anterograde tracing}

Anterograde-tracing was performed to detect axonal regeneration as previously described [21]. Biotinylated dextran amine (BDA; MW,10000; Invitrogen) 10\% w/v in sterile PBS was injected into two sites $(0.4 \mu \mathrm{l} / \mathrm{site})$ of the spinal cord by micropipette. One week later, mice were anesthetized and perfused transcardially with $4 \%$ paraformaldehyde. Thirty-micron sections were incubated with Alexafluor 488-conjugated streptavidin (1: 500; Invitrogen) for $1 \mathrm{~h}$ at room temperature. The images were acquired with a confocal microscope (Olympus, BX51).

\section{Cell culture}

PC12 cells (Cell Storage Center of Wuhan University) were cultured in Dulbecco's modified Eagle's medium and Ham's F-12 (DMEM/F12, Hyclone, USA) supplemented with $10 \%$ horse serum (Hyclone, USA), $5 \%$ fetal bovine serum (FBS) (Hyclone, USA), and 1\% penicillin/ streptomycin (Hyclone, USA). Cultures were maintained at $37{ }^{\circ} \mathrm{C}, 95 \%$ air, and $5 \% \mathrm{CO}_{2}$ in a cell culture incubator (Thermo Fisher Scientific, USA).

\section{Oxygen-glucose deprivation/reoxygenation and drug treatment}

Oxygen-glucose deprivation/reoxygenation (OGD/R) was used to model ischemic injury in vitro, as described previously [22]. Briefly, PC12 cell cultures were washed with ice-cold PBS and culture medium was replaced with glucose-free DMEM (Gibco, USA). Cells were incubated in a hypoxic incubator (Thermo Scientific; USA) at $94 \% \mathrm{~N}_{2}, 5 \% \mathrm{CO}_{2}$, and $1 \% \mathrm{O}_{2}$ for $2 \mathrm{~h}$ at $37{ }^{\circ} \mathrm{C}$. Cells were then returned to standard culture medium and incubation conditions $\left(37{ }^{\circ} \mathrm{C}, 95 \%\right.$ air, and $\left.5 \% \mathrm{CO}_{2}\right)$. The ROS inhibitor, $\mathrm{N}$-acetyl cysteine (NAC, Sigma-Aldrich, USA), was dissolved in sterile $\mathrm{H}_{2} \mathrm{O}$ prior to cellular treatments [23].

\section{Lactate dehydrogenase assay}

PC12 cells were cultured in 96-well plates with or without NAC (at concentrations of $0,10,20,30,50$, or 100 $\mu \mathrm{M})$ for 3,6 , or $24 \mathrm{~h}$. Culture supernatant was collected and analyzed using a commercial lactate dehydrogenase (LDH) kit (Beyotime, China) according to the manufacturer's instructions.

\section{Detection of reactive oxygen species}

To determine reactive oxygen species (ROS) levels in the spinal cord, tissue samples were homogenized in $10 \mathrm{ml} /$ $\mathrm{g}$ of ice-cold PBS as described previously [24]. Homogenates were centrifuged at $10,000 \times g$ for $10 \mathrm{~min}$ at $4{ }^{\circ} \mathrm{C}$. Supernatants were collected and diluted 200-fold in PBS. Then, $100 \mu \mathrm{l}$ of diluted supernatant was mixed with 100 $\mu \mathrm{l}$ of the molecular probe, 20,70-dichlorodihydrofluorescein diacetate (DCFH-DA, $10 \mu \mathrm{M}$; Sigma-Aldrich, USA), and was then transferred to a microplate culture well. To determine PC12 intracellular ROS levels, DCFH-DA $(10 \mu \mathrm{M})$ was added to cells in serum-free medium. Cells and tissue sample supernatants were incubated with DCFH-DA at $37{ }^{\circ} \mathrm{C}$ for $1 \mathrm{~h}$ in the dark. ROS levels were measured at $492 / 520 \mathrm{~nm}$ using a multiplate reader (Synergy HT; Biotech, USA).

\section{Caspase-1 activity}

Caspase-1 activity was measured using the Caspase-1 Activity Assay Kit (RD, K111-100) according to the manufacturer's instructions. Briefly, 2-5 $\times 10^{6}$ cells were lysed in $50-100 \mu \mathrm{l}$ of ice-cold lysis buffer for $10 \mathrm{~min}$ and were then centrifuged. Each specimen was incubated in a 96-well plate with Ac-YVAD-pNA $(200 \mu \mathrm{M})$ at $37{ }^{\circ} \mathrm{C}$ for $2 \mathrm{~h}$. The optical density of each specimen was read on a microplate reader (Synergy HT; Biotech, USA).

\section{Scanning electron microscopy}

PC12 cells were fixed in 2.5\% glutaraldehyde for $3 \mathrm{~h}$ and were then rinsed with PBS. Cells were dehydrated through a graded ethanol series, dried using tertiary butanol, sputter coated with gold, and imaged via scanning electron microscopy.

\section{Flow cytometry}

The PC12 cell apoptotic rate was determined using flow cytometry with an annexin V-FITC/PI apoptosis detection kit (BD Biosciences, 556547) according to the manufacturer's instructions. Briefly, single-cell suspensions were stained with annexin- $\mathrm{V}$ and propidium iodide (PI) at room temperature for $15 \mathrm{~min}$ in the dark and were subjected to flow cytometry (BD Biosciences, San Jose, CA). Data were analyzed using FlowJo (TreeStar, Ashland, OR).

\section{Immunofluorescence}

Immunofluorescent staining was performed as previously described [25]. Briefly, tissue sections or cells were blocked using a $10 \%$ bovine serum albumin solution with $0.25 \%$ triton $\mathrm{X}-100$ for $1 \mathrm{~h}$ at room temperature, 
followed by incubation overnight at $4{ }^{\circ} \mathrm{C}$ with primary antibodies. These primary antibodies included rabbit anti-NeuN (1:500, Millipore), rabbit anti-GFAP (1:500, Cell Signaling Technology), rabbit anti-IBA-1 (1:500, Wako Pure Chemical Industries), goat anti-IBA-1 (1:500, Wako Pure Chemical Industries), mouse anti-GSDMD (1:50, Santa-Cruz), mouse anti-ASC (1:200, Santa-Cruz), mouse anti-caspase-1 (1:200, Santa-Cruz), mouse antiNLRP3 (1:200, AdipoGen), rabbit anti-Hv1 (1:50, Sigma), and mouse anti-8-OHdG (1:200, Abcam). Next, samples were washed in PBS and incubated at room temperature for $1 \mathrm{~h}$ with the appropriate secondary antibodies, as follows: FITC-conjugated goat anti-mouse immunoglobulin G (IgG), Cy3-conjugated goat anti-rabbit IgG, Cy3conjugated rabbit anti goat IgG, Cy3-conjugated rabbit anti-rat IgG, and 488-conjugated donkey anti-goat IgG (Jackson ImmunoResearch, West Grove, PA, USA). Finally, sections were stained with 4,6-diamidino-2-phenylindole (DAPI). Samples were imaged using a confocal microscope (Olympus, BX51).

\section{TUNEL assay}

Apoptosis was detected using the In-Situ Cell Death Detection Kit (TUNEL fluorescence FITC kit, Roche) according to the manufacturer's instructions. TUNEL staining was performed with fluorescein-dUTP for detection of apoptotic cell nuclei and DAPI for staining of cellular nuclei. The number of TUNEL-positive cells was analyzed via confocal microscopy (Olympus, BX51).

\section{Western blotting}

Tissue samples or PC12 cells were lysed in total protein lysis buffer and protein concentrations were determined using a BCA protein kit (Beyotime, China). Proteins (30 $\mu \mathrm{g})$ were separated by sodium dodecyl sulfatepolyacrylamide gels (SDS-PAGE) and were transferred to nitrocellulose filters (NCs) or polyvinylidenedifluoride (PVDF) membranes. Membranes were blocked for $1 \mathrm{~h}$ at room temperature using $5 \%$ non-fat milk in Tris-buffered saline containing $0.1 \%$ Tween-20, then incubated overnight at $4{ }^{\circ} \mathrm{C}$ with primary antibodies. The following primary antibodies were used: rabbit antiGSDMD (1:1000, Cell Signaling Technology), mouse anti-Caspase-1 (1:500, AdipoGen), mouse anti-NLRP3 (1:1000, AdipoGen), rabbit anti-ASC (1:1000, Cell Signaling Technology), rabbit anti-IL-18 (1:1000, Abclonal), rabbit anti-Hv1 (1:1000, Sigma), rabbit anti-NOS2 (1: 1000; Abclonal), mouse anti-NF-L and anti-NF-H (1: 1000; Cell Signaling Technology), rabbit anti-TUJ1 (1: 1000, Abcam), mouse anti-MAG (1:1000, Santa Cruz), rat anti-MBP (1:1000, Millipore), rabbit anti- $\beta$-actin (1: 1000 , Servicebio), and rabbit anti-GAPDH (1:1000, Servicebio). Membranes were subsequently incubated with horseradish peroxidase-labeled anti-rabbit, anti-mouse, or anti-rat secondary antibodies (1:5000, Servicebio). Bands were visualized using a Bio-Rad Chemidoc XRS+ imaging system with enhanced chemiluminescent kits (Advansta). Protein levels were determined based on O.D. value using ImageJ software. Protein expression levels were normalized to $\beta$-actin or GAPDH internal controls.

\section{Real-time and semi-quantitative PCR}

Spinal cord tissue samples were collected at 1, 3, or 7 days ( $n=5$ for each time point) after surgery and total RNA was isolated using Trizol reagent (Invitrogen). Next, cDNA was prepared using a ReverTra Ace qPCR RT Kit (TOYOBO, Japan). Quantitative real-time PCR (qRT-PCR) was performed using a Real-Time PCR system (BioRad) and SYBR Green PCR Master Mix (TOYOBO, Japan). Then, mRNA expression levels were normalized to those of an endogenous reference gene, glyceraldehyde 3-phosphate dehydrogenase (GAPDH). Semi-quantitative PCR was performed using Quick Taq HS Dye Mix (Japan). PCR products were electrophoresed on a $2 \%$ agarose gel and were visualized with GelRed (Biotium; Hayward, CA) and a Gene Genius BioImaging System (Syngene; Cambridge, UK). The following primers were used: interleukin-18 (IL-18) (forward: 5'GGCCGACTTCACTGTACAACCG3', reverse: 5'GG TCACAGCCAGTCCTCTTACTTC3'), GAPDH (forward: 5'GGTTGTCTCCTGCGACTTCA3', reverse: TG GTCCAGGGTTTCTTACTCC), Hv1 (forward: $5^{\prime}$-GAG ATCCATCTGCCTCCGTTATGAGTG-3', reverse: $5^{\prime}$ CTGTGTCTCCCTGTGGCTGAG- ${ }^{\prime}$ ), and $\beta$-Geo-R (5' -GACAGTATCGGCCTCAGGAAGATCG-3').

\section{Statistical analysis}

All data are presented as the mean \pm standard error of the mean (SEM). Data were evaluated by one-way or two-way analyses of variance (ANOVAs) with Tukey's post-hoc tests, when necessary. A value of $p<0.05$ was considered statistically significant. Statistical analyses were performed using GraphPad Prism 6 (GraphPad Software, Inc., La Jolla, CA).

\section{Results}

\section{Confirmation of Hv1 gene knockout}

PCR analysis of genomic DNA revealed a 580-bp band in samples from WT but not $\mathrm{Hv}^{-1-}$ (KO) mice (Fig. 1a). $\mathrm{Hv1}$ protein was not detected in Western-blot analysis of KO mice (Fig. 1b). Immunofluorescent analysis clearly showed Hv1 expression in IBA-1-positive microglia from WT mice, but Hv1 immunoreactivity was rarely detected in microglia from $\mathrm{KO}$ mice (Fig. 1c). 


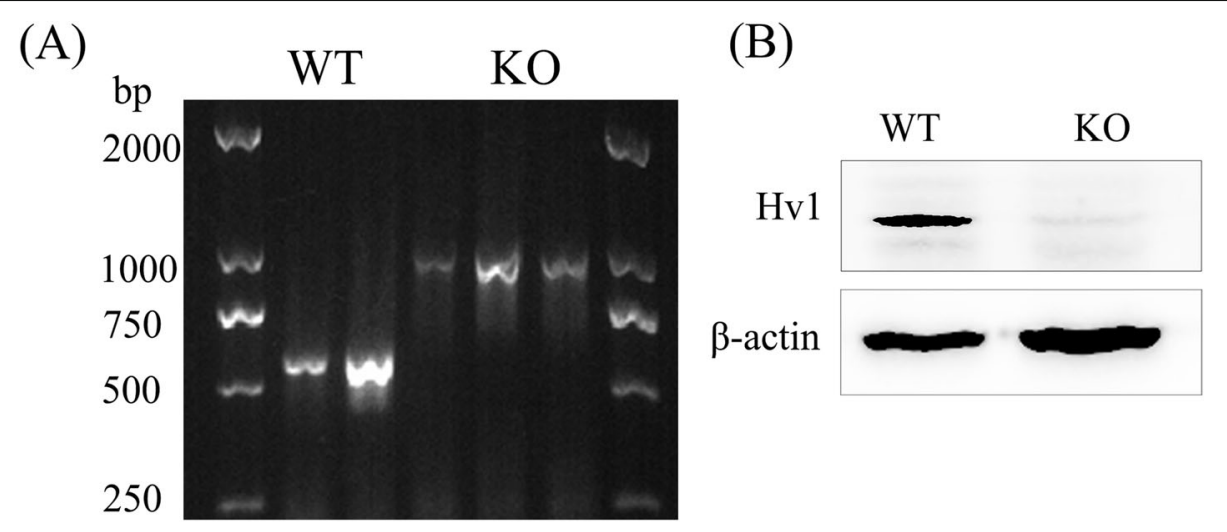

(C)

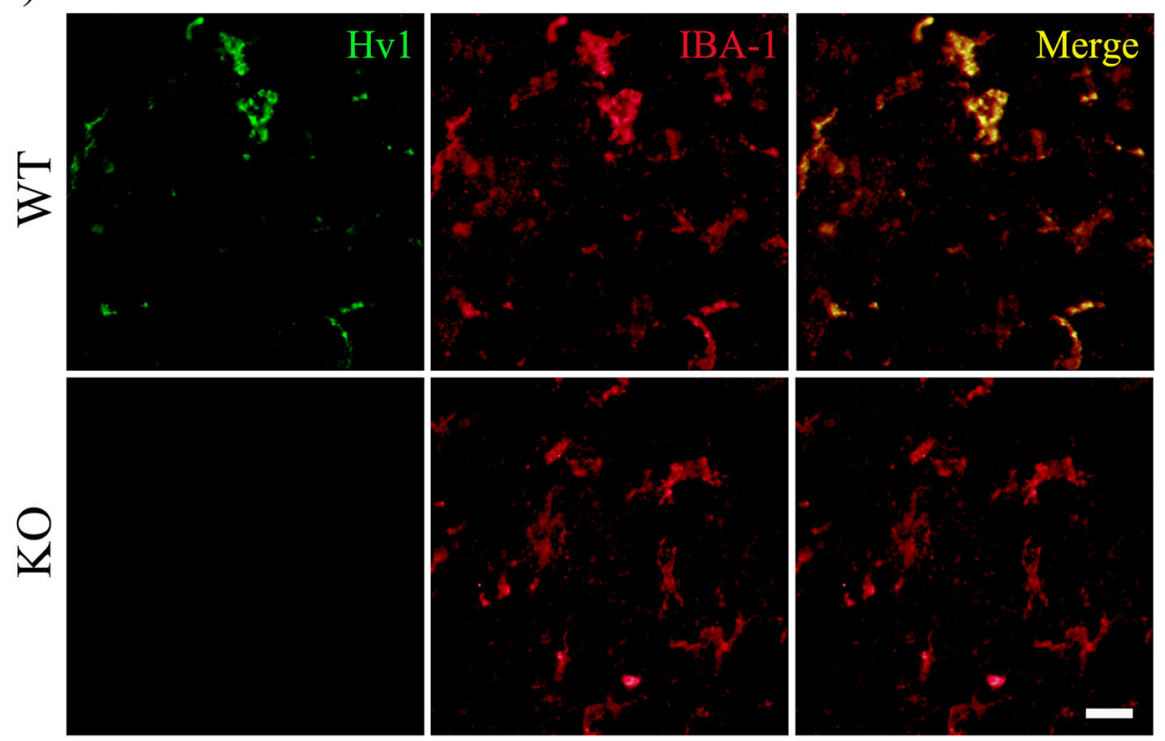

Fig. 1 Genotype identification in $\mathrm{Hv}^{-1-}$ mice. a Tail DNA analyzed by PCR. $\mathbf{b} \mathrm{Hv} 1$ protein deficiency confirmed by Western blotting. $\mathbf{c}$ Expression of Hv1 in microglia assessed by double-immunofluorescence labeling with Hv1 (green) and the microglial marker, IBA-1 (red; scale bar, 50 um). Hv1 was co-localized with the microglial marker, IBA-1, in wild-type (WT) mice. In contrast, Hv1 immunofluorescent signal was rarely observed in $\mathrm{HV}^{-1-}(\mathrm{KO})$ mice

\section{Distinct spatiotemporal patterns of neuronal pyroptosis} and apoptosis following $\mathrm{SCl}$; microglial Hv1 deficiency inhibits neuronal pyroptosis and apoptosis

Increasing evidence indicates acute-phase neuronal pyroptosis and apoptosis participation in the neuropathology of SCI [14]. First, a double-labeling for GSDMD and TUNEL, which are markers of pyroptosis and apoptosis respectively, was performed (Fig. 2a). We found distinct spatial patterns, with TUNEL-positive cells mainly located closer to the injury core than GSDMDpositive cells (Fig. 2a). To better understand the temporal distribution of pyroptotic cells after SCI, we performed a double-labeling of GSDMD and NeuN (Fig. $2 b)$. We found that the frequency of neurons $\left(\mathrm{NeuN}^{+}\right.$ cells) expressing GSDMD was significantly elevated in WT mice after SCI, peaking at 3 days $(p<0.01)$ (Fig. 2c), demonstrating SCI-linked neuronal pyroptosis. To investigate the temporal pattern of neuronal apoptosis, we used a TUNEL assay and observed a significant increase in neuronal apoptosis that peaked at 1-day post-SCI in WT mice $(p<0.01)$ (Fig. $2 \mathrm{~d}$, e). Interestingly, the duration of neuronal pyroptosis exceeded that of apoptosis after SCI (Fig. 2c, e).

We further investigated the effects of Hv1 deficiency on neuronal pyroptosis and apoptosis after SCI by comparing the frequencies of neurons $(\mathrm{NeuN}+)$ doublelabeled by GSDMD or TUNEL in KO vs. WT SCI mice. The numbers of GSDMD-positive (assayed on days 1, 3, and 7 post-treatment; all $p<0.01$ ) and TUNEL-positive (assayed on days 1 and 3; $p<0.01, p<0.05$, respectively) neurons were significantly downregulated in $\mathrm{KO}$ SCI mice (Fig. 2c, e). Consistent with these findings, protein 


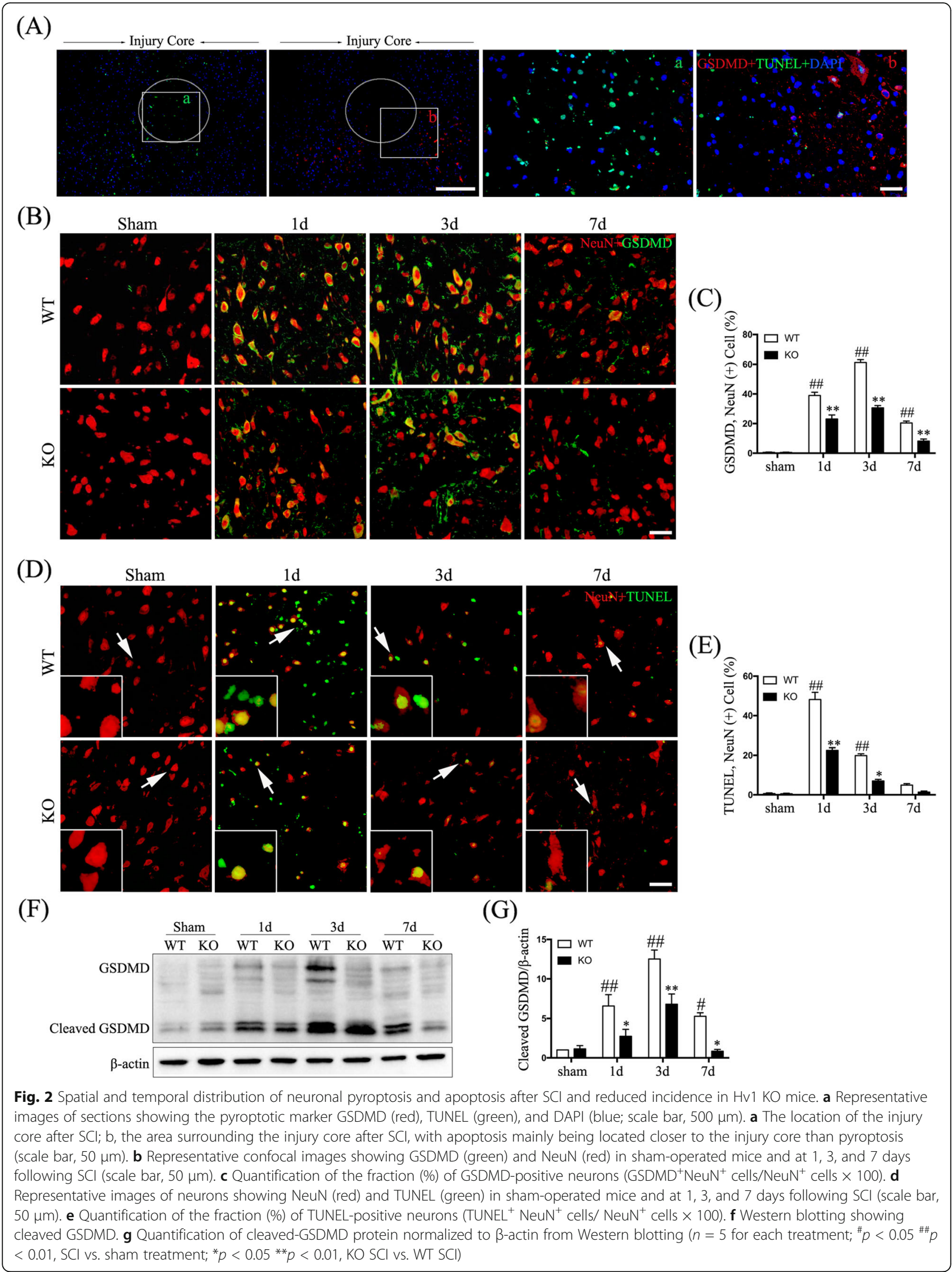


levels of cleaved GSDMD, as determined by Westernblot analysis, were significantly decreased in $\mathrm{KO}$ mice at 1,3 , and 7 days after SCI $(p<0.05$; Fig. 2 f, g).

\section{Hv1 deficiency inhibits activation of the NLRP3 inflammasome}

Upon activation, the NLRP3 inflammasome promotes cleavage of pro-caspase- 1 to caspase- $1 \mathrm{p} 20$, which then cleaves the pyroptotic factor, GSDMD [26]. We observed differential neuronal upregulation of NLRP3, caspase-1, and ASC proximal to the injury site after SCI in WT compared to that in sham-operated mice (Fig. 3a-c). Hv1 KO mice had lower numbers of NLRP3-positive neurons (at 1,3 , and 7 days; all $p<0.01$ ), caspase-1positive neurons (at 1,3 , and 7 days; $p<0.05$ ), and ASC-positive neurons (at 1, 3, and 7 days; $p<0.01$ ) (Fig. $3 \mathrm{~d}-\mathrm{f}$ ). Furthermore, we analyzed NLRP3, ASC, and caspase-1 p20 protein levels via Western blotting (Fig. 3a). At 1 and 3 days following SCI, caspase- 1 p20 and ASC levels were significantly downregulated in $\mathrm{KO}$ mice compared to those in WT mice $(p<0.05)$, and NLRP3 levels in $\mathrm{KO}$ mice were strikingly lower at both 3 and 7 days following SCI $(p<0.05)$ (Fig. 3h-j). Importantly, NLRP3 inflammasome activation has also been reported to promote maturation and release of IL-18 [27]. As shown in Fig. $3 g$ and k, expression of IL-18 protein was upregulated in WT animals at 1 day after SCI compared to that of sham-operated mice and was comparatively decreased in KO mice (all $p<0.01$ ). Next, qRT-PCR analysis confirmed that IL-18 upregulation was significantly increased in WT vs. KO mice at 1 day after SCI $(p<0.01)$ (Fig. 3l). Hv1 deficiency therefore significantly attenuated activation of the NLRP3 inflammasome, which is consistent with the effect of Hv1 deficiency on neuronal pyroptosis.

\section{Hv1 deficiency attenuates ROS production}

In mouse models, secondary damage following SCI is closely linked to excessive microglial ROS generation [28]. We used an 8-OHG-specific antibody to detect oxidized nucleic acids caused by cellular ROS [29]. 8-OHG immunoreactivity was mainly localized in microglia (IBA- $1^{+}$cells) and the percentage of 8-OHG-positive microglia was significantly elevated in the boundary zone of the injury core after SCI compared to that in shamoperated mice (Fig. 4a). Furthermore, the fraction of 8OHG-positive microglia after SCI was markedly lower in $\mathrm{KO}$ mice compared to that in WT mice $(p<0.01)$ (Fig. $4 \mathrm{~b})$. We further observed increased ROS levels in WT mice at both 3 and 7 days after SCI compared to those in sham-operated mice $(p<0.01)$, while SCI elicited lower ROS generation in $\mathrm{Hv1}^{-/-}$mice compared to that in WT mice $(p<0.05)$ (Fig. 4c). Finally, Western-blot analysis showed that NOS2 protein levels were significantly lower in $\mathrm{KO}$ mice compared to those in WT mice at 1 and 7 days after SCI $(p<0.01)$ (Fig. $4 d$, e). These findings suggest an important role of microglial Hv1 in ROS generation after SCI.

\section{ROS regulates pyroptosis in PC12 cells}

To directly address whether Hv1 regulation of ROS generation after SCI is relevant to neuronal pyroptosis and apoptosis, we next employed a PC12-cell OGD/R oxidative stress model in vitro. We first measured cellular oxidative stress using DCFH-DA and observed significantly elevated ROS levels after OGD/R $(p<0.01)$ (Fig. 5a). To clarify the relationship between ROS and neuronal pyroptosis, we used the ROS scavenger, NAC. We first performed LDH assays to measure NACinduced cytotoxicity in PC12 cells. As shown in Fig. 5a, NAC elicited dose- and time-dependent release of $\mathrm{LDH}$. We also found that $30 \mu \mathrm{M}$ of NAC significantly reduced ROS levels in the OGD/R model $(p<0.05)$. Therefore, we used a $30 \mu \mathrm{M}$ concentration of NAC to suppress ROS generation in subsequent experiments.

Pyroptosis is characterized by cellular swelling and the emergence of large bubbles from the plasma membrane [30]. Consistent with these published observations, using scanning electron microscopy, we observed these morphological characteristics in $\mathrm{PC} 12$ cells undergoing OGD/R (Fig. 5b). To determine whether PC12 cell pyroptosis was regulated by ROS, we compared GSDMD expression by immunofluorescence (IF) and Westernblot analyses among untreated control, OGD/R, and OGD/R + NAC-treated cells (Fig. 5c, d). Cleaved GSDM $\mathrm{D}$ protein was markedly upregulated in PC12 cells at 6 and $24 \mathrm{~h}$ after OGD/R and was inhibited by NAC ( $p<$ 0.05 ) (Fig. 5e, f). ROS therefore contributed to PC12 cell pyroptosis in our OGD/R model in vitro.

\section{ROS promotes PC12 cell apoptosis}

To further investigate temporal distinctions in neuronal pyroptosis and apoptosis, as observed following SCI, we measured PC12 cell apoptosis using flow cytometry with annexin V/PI and TUNEL staining (Fig. 6a, b). Apoptosis was significantly increased at 3 and $6 \mathrm{~h}$ after OGD/ $\mathrm{R}(p<0.05)$ (Fig. 6c, d), which was earlier than observations for pyroptosis. We also found that $\mathrm{PC} 12$ cell apoptosis was reduced in $\mathrm{OGD} / \mathrm{R}$-treated cells exposed to NAC $(p<0.05)$ (Fig. 6c, d).

\section{ROS scavenger rescues activation of the NLRP3 inflammasome in $\mathrm{PC} 12$ cells after $\mathrm{OGD} / \mathrm{R}$}

We used the OGD/R PC12 cell model to investigate the relationship between ROS and the NLRP3 inflammasome pathway. Activation of the NLRP3 inflammasome (consisting of NLRP3, ASC, and pro-caspase1) was determined by Western blotting (Fig. 7a). Levels of NLRP3, 


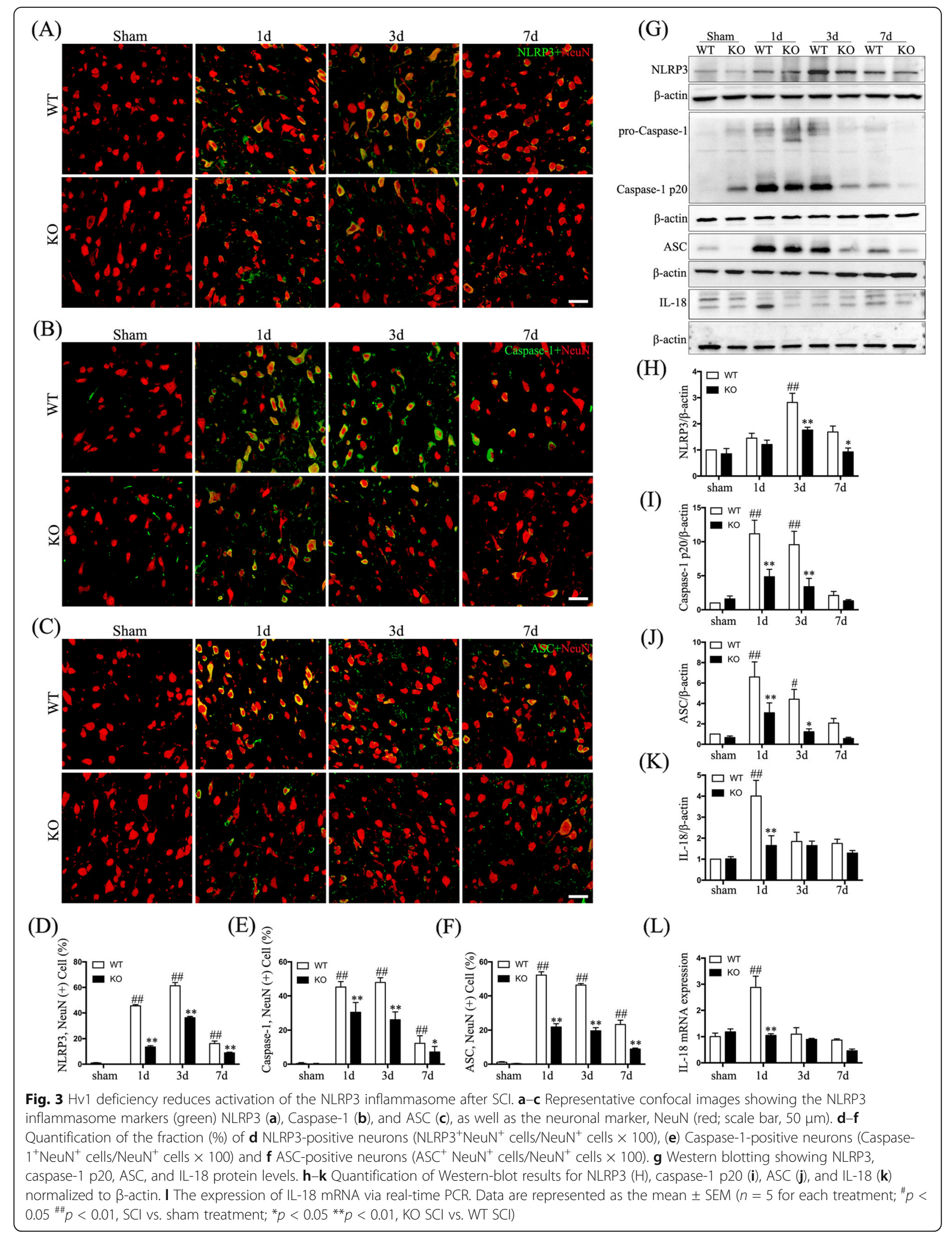




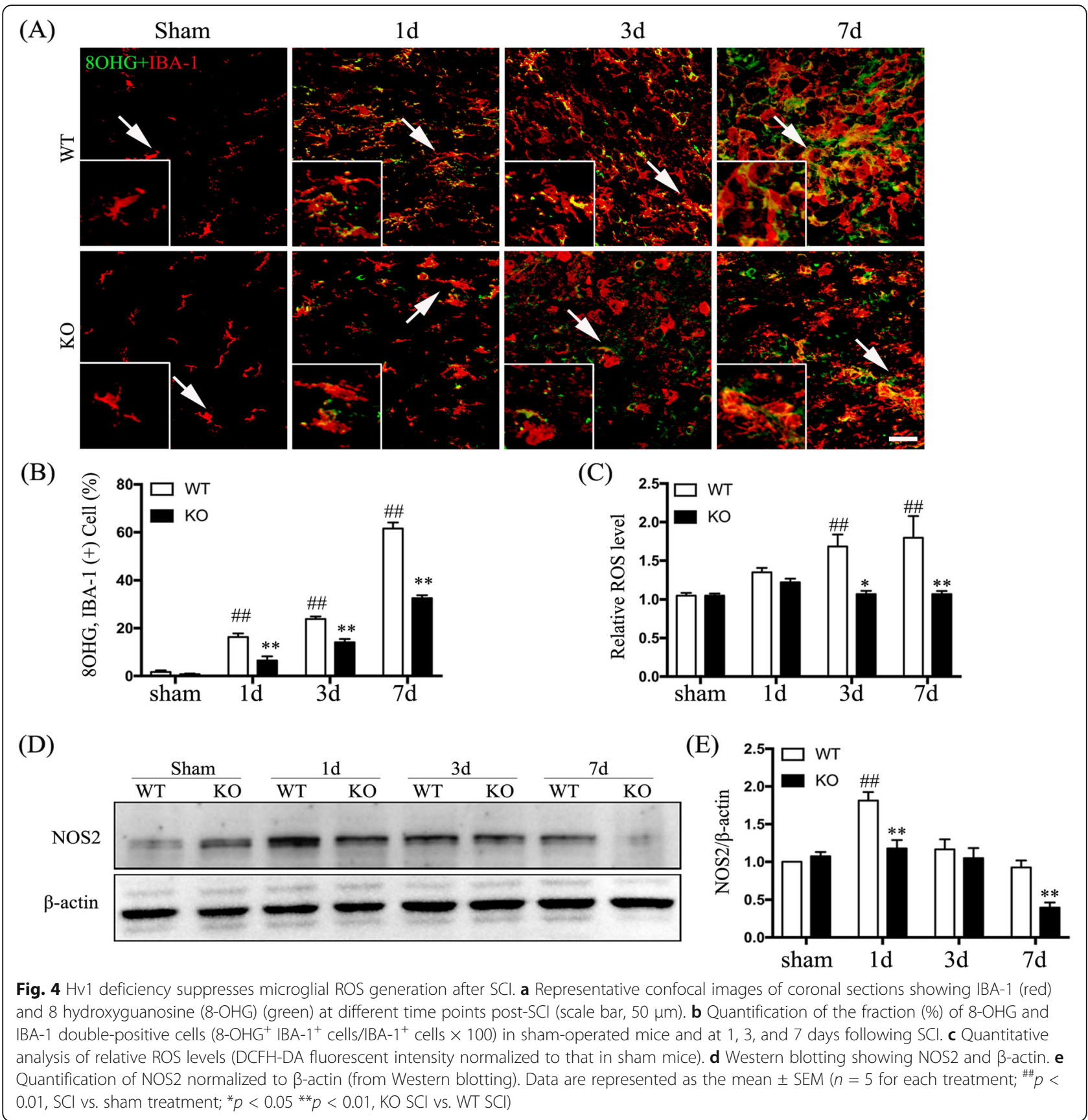

ASC, and caspase-1 p20 were significantly upregulated after OGD/R $(p<0.05)$ (Fig. 7b-d), while levels of NLRP3, ASC, and cleaved caspase-1 were significantly downregulated by the addition of NAC $(p<0.05)$ (Fig. $7 \mathrm{~b}-\mathrm{d})$. Caspase- 1 activity was directly measured and shown to be enhanced at $6 \mathrm{~h}$ after OGD/R but was inhibited by addition of NAC $(p<0.05)$ (Fig. 7e). We also found that upregulation of IL-18 protein level at $6 \mathrm{~h}$ after OGD/R was suppressed by NAC $(p<0.01)$ (Fig. 7a, f). These results suggest that ROS is essential for activation of the NLRP3 inflammasome pathway in PC12 cells in the OGD/R model.

Hv1 deficiency promotes axonal regeneration and the recovery of motor functions after $\mathrm{SCl}$

To further investigate the effects of Hv1 deficiency on lesion volumes and axonal recovery following SCI, we performed LFB staining, GFAP staining, and anterograde tracing (Fig. 8a, b). After 14 and 28 days after SCI, LFB staining demonstrated that lesion volumes were smaller 


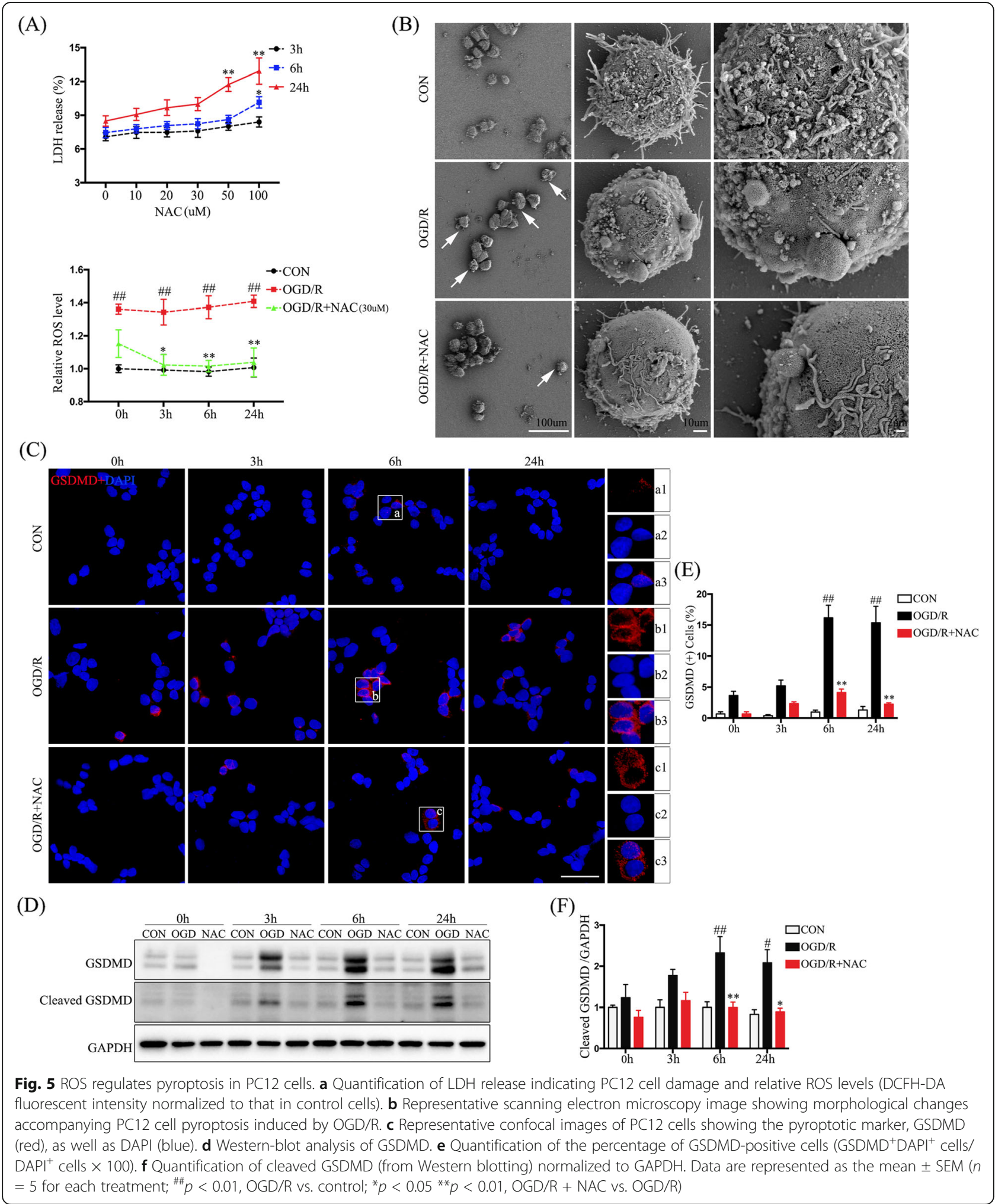

in $\mathrm{Hv1}^{-1-}$ mice than that in WT mice (Fig. 8a). As shown in Fig. 8b, many integrated BDA-labeled axons were observed in sham-operated groups. After 28 days after SCI, few BDA-labeled axons were visualized in the caudal side of the injury in WT groups; however, in $\mathrm{Hv}^{-/-}$mice, some axonal sprouts passed through astrocyte scar borders and extended into the spared grey matter. Then, we further assessed levels of the axonal 


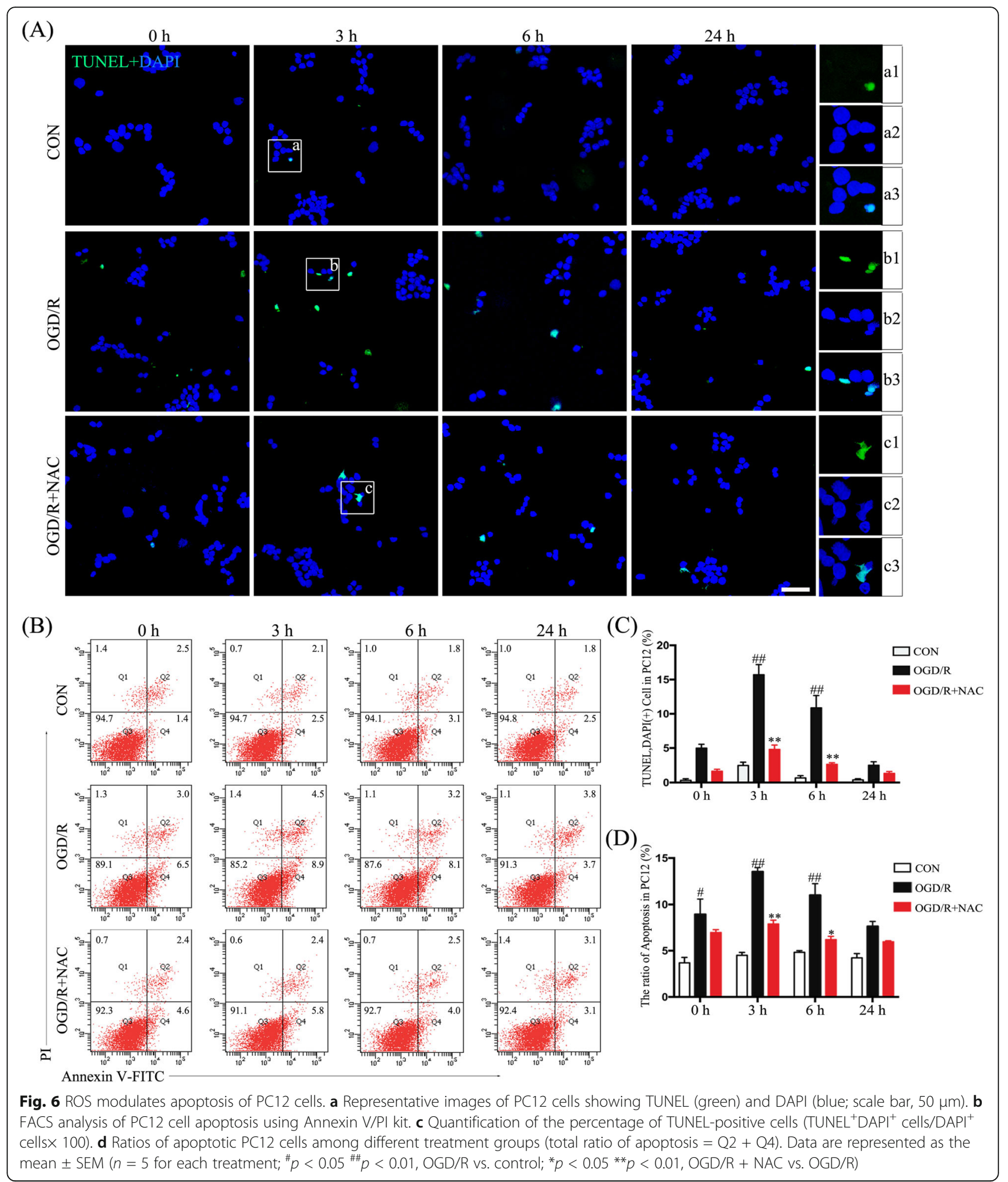

markers, neurofilament-L (NF-L) and neurofilament-H (NF-H), and the neuronal-specific marker, BIII-tubulin (TUJ1), via Western blotting (Fig. 8c). In $\mathrm{Hv}^{-1-}$ mice, NF-H and TUJ1 were increased at day 14 , while NF-L was increased at 14 and 28 days after SCI $(p<0.05)$ (Fig. 8d-f). Myelin-associated glycoprotein (MAG) is key to maintaining axon-glial integrity and myelin basic protein (MBP) is a general myelin marker. MAG and MBP levels were significantly increased at 28 and 14 days following $\mathrm{SCI}$ in $\mathrm{KO}$ mice, respectively, compared to those in WT 


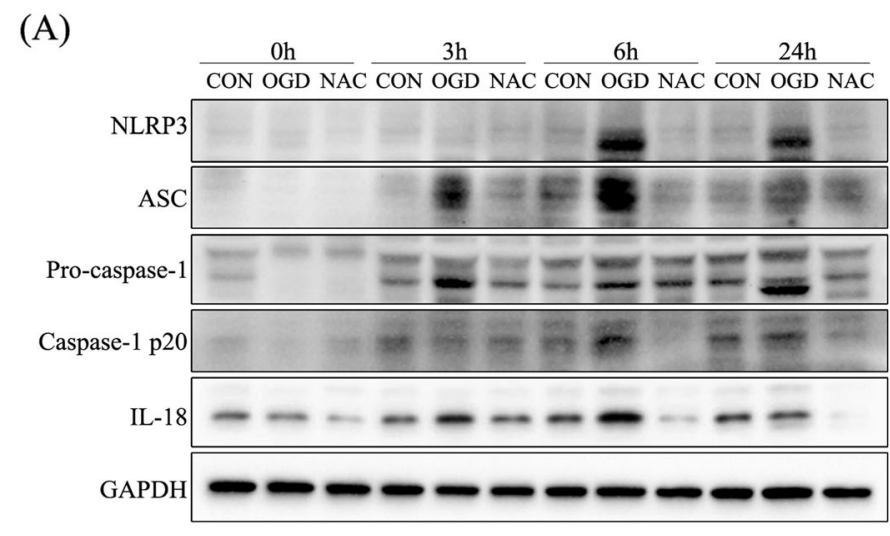

(D)

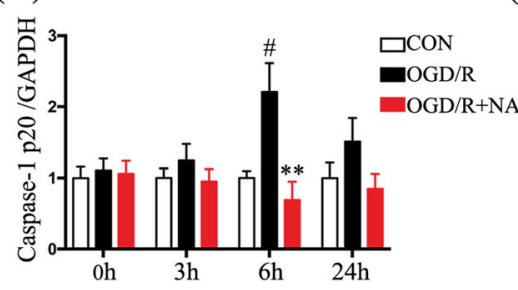

(E)

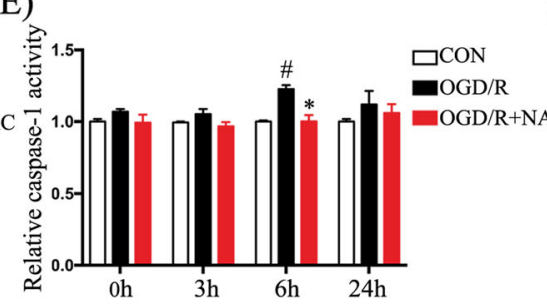

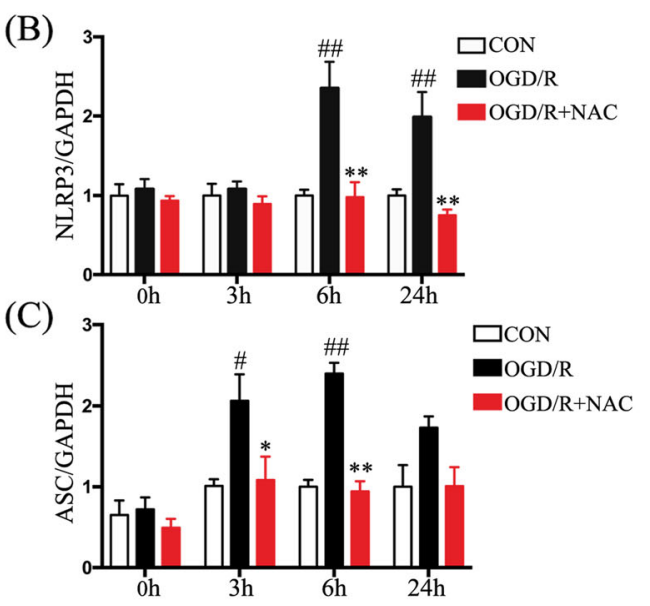

(F)

Fig. 7 A ROS scavenger rescues ROS-mediated NLRP3 inflammasome activation. a Western blotting showing NLRP3, ASC, caspase-1 p20, and IL18 levels. b-d Quantification of Western-blot analysis for NLRP3 (b), ASC (c), and caspase-1 p20 (d) normalized to GAPDH. e Relative caspase-1 activity after different treatments. $\mathbf{f}$ Quantification of Western-blot analysis for IL-18 normalized to GAPDH. Data are represented as the mean \pm SEM ( $n=5$ for each treatment; ${ }^{\#} p<0.05^{\# \#} p<0.01, \mathrm{OGD} / \mathrm{R}$ vs. control; ${ }^{*} p<0.05{ }^{* *} p<0.01, \mathrm{OGD} / \mathrm{R}+\mathrm{NAC}$ vs. OGD/R)

mice $(p<0.05)$ (Fig. 8g, h). Motor function recovery was evaluated using the BMS scoring system [20]. BMS scores were markedly better for $\mathrm{KO}$ mice compared to those for WT mice at 14 and 28 days after SCI $(p<$ 0.05) (Fig. 8i). These observations are consistent with Hv1 deficiency promoting axonal regeneration and recovery of motor function after SCI.

\section{Discussion}

The present study found distinct spatial and temporal patterns of neuronal pyroptosis and apoptosis after SCI and showed that Hv1 deletion significantly attenuated NLRP3-inflammasome-mediated neuronal pyroptosis and apoptosis as well as facilitated myelin-axon regeneration paralleled by improved motor function. These findings suggest that the effects on ROS-mediated modulation of NLRP3 inflammasome activation underlie protection against neuronal damage from Hv1 deficiency. To our knowledge, the present study is the first to elucidate spatial and temporal differences in neuronal pyroptosis and apoptosis during early-stage SCI. Moreover, this work is the first to reveal the underlying mechanism by which Hv1 deficiency attenuates neuronal pyroptosis after SCI.

Apoptosis has long been recognized as the predominant in a wide variety of neurological conditions [31].
Recently, findings found that another special death mechanism different from apoptosis participated in the CNS pathology, which has been named as pyroptosis in 2001 [32, 33]. Pyroptosis is characterized by cellular swelling [30], formation of 10-14-nm GSDMD pores [34], caspase-1-dependent cell death, and release of inflammatory cytokines such as IL-18 [33]. More recently, neuronal pyroptosis-a gasdermin-mediated programmed necrotic cell death-has been reported to be linked to traumatic brain injury [33], cerebral ische$\mathrm{mia} /$ reperfusion injury [25], and SCI [14]. In the present study, we showed that neuronal expression levels of the pyroptosis marker GSDMD and the inflammatory factor IL-18 were significantly upregulated in vivo SCI model in mice and in vitro OGD/R model in neurons mimicking SCI, which are consistent with the previous opinion that neuronal pyroptosis contributed to neuroinflammation after SCI [33]. Accumulating studies showed that apoptosis and pyroptosis could both occur in neuron during the pathology of CNS diseases [25, 33]. However, few researches further focused on the spatiotemporal patterns of neuronal pyroptosis and apoptosis. Here, for the first time, we reported that the peak of neuronal apoptosis prior to that of neuronal pyroptosis and the duration of pyroptosis was much longer than that of apoptosis in vitro and in vivo experiments. These results 


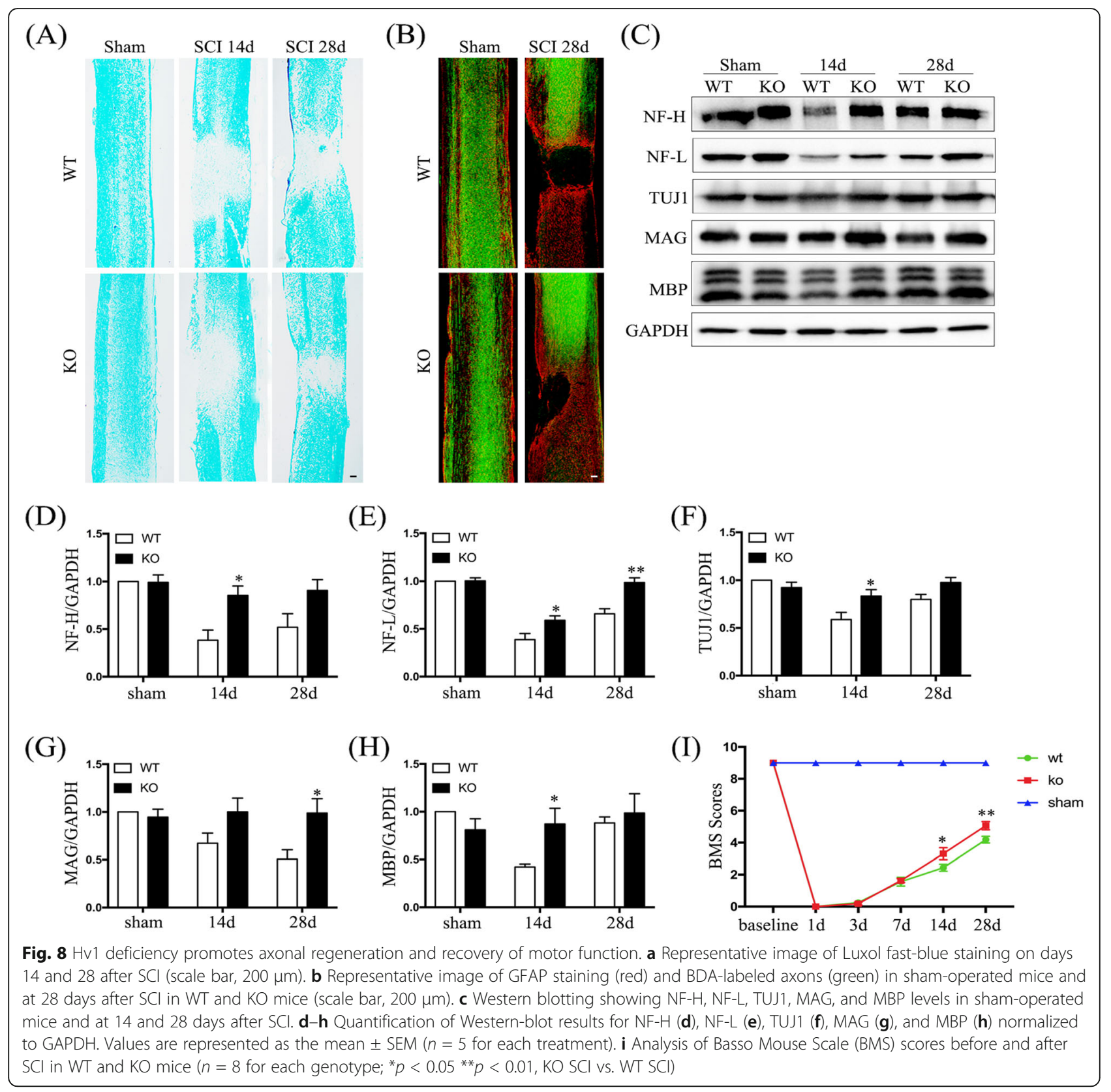

suggest that in contrast to apoptosis, timely intervention to inhibit neuronal pyroptosis following SCI is feasible. Thus, identifying a means of regulating neuronal pyroptosis after SCI is potentially valuable.

Hv1 channel has been reported to regulate neuronal death in the pathogenesis of ischemic stroke [8]. Our present study also showed that neuronal apoptosis following SCI was reduced in $\mathrm{Hv}^{-/-}$mice relative to that in WT mice. However, information on microglial Hv1-mediated neuronal pyroptosis remains unclear. Interestingly, our study is the first to discover that the expression of pyroptosis in neurons was lower in $\mathrm{Hv}^{-/-}$ mice compared with that in WT mice after SCI.
Microglia and macrophages, the first cells to be recruited in response to injury, are the most important modulators of secondary damage after SCI [35] and activated microglia contribute to ROS generation [36]. Hv1 channel, functionally expressed in microglia, has been reported to induce NADPH oxidase (NOX)-mediated ROS generation [8]. In the current study, we observed upregulation of microglial ROS generation after SCI, which was significantly attenuated in $\mathrm{Hv}^{-/-}$mice in the acute period after SCI. This finding is consistent with our previous report that microglial Hv1 deficiency could attenuate ROS level [10]. Emerging evidence showed that ROS signaling was the key in the initiation of pyroptotic death 
involving diverse diseases [37, 38]. It was proposed that pyroptosis in hemorrhagic shock and resuscitation was downregulated through reducing ROS production by mitochondria [37]. In another study, ischemiareprerfusion-induced pyroptosis remarkably relied on ROS which was significantly inhibited by ROS scavenger NAC [38]. In line with these previous researches, our in vitro data further showed that ROS-induced pyroptosis by OGD/R model in neurons was obviously rescued by NAC. Therefore, we reasonably believe that microglial Hv1 channel exacerbated neuronal pyroptosis through enhancing ROS production after SCI.

Unlike apoptosis, pyroptosis is an inflammatory cell death requiring the activation of caspase-1 [39]. Caspase-1 activation is modulated by protein complexes termed inflammasomes. Inflammasomes can be divided into different types, such as NLRP1, NLRP2, NLRP3, NLRC4, NLRP6, NLRP7, and AIM2 [14]. Among these inflammasomes, NLRP3 inflammasome expression is markedly upregulated following SCI, thus playing an important role in spinal cord tissue after SCI [40]. Furthermore, Zendedel et al. [41] found that NLRP3 was expressed in neurons, microglia, and astrocytes, and that neurons represented the major source of NLRP3. Here, we showed that the expressions of NLRP3, ASC, and caspase-1 p20 in neurons were significantly increased after $\mathrm{SCI}$ in mice, the findings of which are consistent with those of a previous study [14]. In addition, NLRP3, ASC, and caspase-1 levels were lower in $\mathrm{Hv}^{-/-}$mice, suggesting that microglial $\mathrm{Hv1}$ is a potential target for modulating the NLRP3 inflammasome. NLRP3 is triggered in response to diverse stimuli including microparticles, pore-forming toxins, ATP, and ROS [42]. ROS acts as a second messenger that plays a significant role in NLRP3 inflammasome activation [43]. The activation of NLRP3 recruits ASC and promotes the activation of caspase-1, thus processing cytokines to their active forms to ultimately induce pyroptotic death [27]. We detected OGD/R activation of the NLRP3 inflammasome pathway in neurons in vitro, which again was inhibited by NAC, thus revealing a key role of ROS in this process. These results suggest that microglial Hv1induced ROS generation regulates NLRP3-inflammasomemediated pyroptosis after SCI.

Neuronal death in the injured area following SCI may contribute to disruption of neural circuitry, ultimately leading to functional impairment [44]. Hence, inhibition of neuronal delayed death is critical to promoting axonal regeneration after SCI [45]. Additionally, reducing neuroinflammation may create a favorable microenvironment for neuronal and myelin repair [46]. Collectively, our findings suggest that inhibition of Hv1 can promote myelin/axonal regeneration and concomitantly improve motor function after SCI. Based on our current results, we propose that microglial Hv1 deficiency may serve as a promising strategy to improve $\mathrm{SCI}$ outcomes.

\section{Conclusions}

In summary, our study provides novel insight into the spatiotemporal patterns of neuronal apoptosis and pyroptosis after SCI, and reveals a potential efficacy of targeted inhibition of microglial Hv1 to prevent neuronal pyroptosis. Accordingly, the development of therapeutic interventions designed to inhibit microglial Hv1 after SCI may be beneficial in afflicted patients.

\begin{abstract}
Abbreviations
SCl: Spinal cord injury; ROS: Reactive oxygen species; WT: Wild-type; KO: Knockout; GSDMD: Gasdermin D; NLRP3: Nod-like receptor 3; ASC: Apoptosis-associated speck-like protein; BMS: Basso mouse scale; PBS: Phosphate-buffered saline; PFA: Paraformaldehyde; LFB: Luxol fast blue; BDA: Biotinylated dextran amine; TUNEL: Terminal dexynucleotidyltransferasemediated dUTP nick end labeling; DMEM/F12: Dulbecco's modified eagle's medium and Ham's F-12; FBS: Fetal bovine serum; OGD/R: Oxygen-glucose deprivation/reoxygenation; NAC: N-acetyl cysteine; LDH: Lactate dehydrogenase; DCFH-DA: Dichlorodihydrofluorescein diacetate; SEM: Standard error of the mean; PI: Propidium iodide;

IF: Immunofluorescence; DAPI: 4,6-Diamidino-2-phenylindole; SDSPAGE: Sodium dodecyl sulfate-polyacrylamide gel; NC: Nitrocellulose filter; PVDF: Polyvinylidenedifluoride; qRT-PCR: Quantitative real-time PCR; IL18: Interleukin-18; ANOVA: Analysis of variance; NF-L: Neurofilament-L; NFH: Neurofilament-H; TUJ1: Blll-tubulin; MAG: Myelin-associated glycoprotein; MBP: Myelin basic protein
\end{abstract}

\section{Acknowledgements}

We thank Prof. Long-Jun Wu (Department of Cell Biology and Neuroscience, Rutgers University) for kindly providing the $\mathrm{Hv}^{-/-}$mice.

\section{Authors' contributions}

$\mathrm{XL}$ and $\mathrm{WW}$ designed the experiments and reviewed the paper. $\mathrm{XFL}, \mathrm{ZYY}$, and WFZ carried out the experiments. XFL, PC, JL, and FFD participated in the data acquisition and analysis. XFL, ZYY, MHW, and MJX wrote the manuscript and revised the manuscript. All authors read and approved the final manuscript.

\section{Funding}

This work was supported by the National Nature Science Foundation of China (81771341 to X. Luo), the National Major Scientific and Technological Special Project for "Significant New Drugs Development" (2017ZXX9304022 to W. Wang.), and Nature Science Foundation of Hubei (2017CFB705 to ZY. $\mathrm{Yu})$.

\section{Availability of data and materials}

Information about the experimental methods, animal model, and data supporting the conclusions of current study are included within the article.

\section{Ethics approval and consent to participate}

All animal procedures were approved by the Institutional Animal Care and Use Committee at Tongji Medical College, Huazhong University of Science and Technology.

\section{Consent for publication}

Yes.

\section{Competing interests}

All authors declare that they have no competing interests.

Received: 3 August 2019 Accepted: 25 August 2020

Published online: 05 September 2020

\section{References}

1. Ulndreaj A, Badner A, Fehlings MG. Promising neuroprotective strategies for traumatic spinal cord injury with a focus on the differential effects among anatomical levels of injury. F1000Res. 2017;6:1907. 
2. Dai W, Wang X, Teng H, Li C, Wang B, Wang J. Celastrol inhibits microglial pyroptosis and attenuates inflammatory reaction in acute spinal cord injury rats. Int Immunopharmacol. 2019;66:215-23.

3. Mortezaee K, Khanlarkhani N, Beyer C, Zendedel A. Inflammasome: its role in traumatic brain and spinal cord injury. J Cell Physiol. 2018;233:5160-9.

4. Karova K, Wainwright JV, Machova-Urdzikova L, Pisal RV, Schmidt M, Jendelova P, Jhanwar-Uniyal M. Transplantation of neural precursors generated from spinal progenitor cells reduces inflammation in spinal cord injury via NF-kappaB pathway inhibition. J Neuroinflammation. 2019;16:12.

5. Ma L, Mu Y, Zhang Z, Sun Q. Eugenol promotes functional recovery and alleviates inflammation, oxidative stress, and neural apoptosis in a rat model of spinal cord injury. Restor Neurol Neurosci. 2018;36:659-68.

6. Loane DJ, Kumar A. Microglia in the TBI brain: the good, the bad, and the dysregulated. Exp Neurol. 2016;275(Pt 3):316-27.

7. Qiu F, Chamberlin A, Watkins BM, lonescu A, Perez ME, Barro-Soria R, Gonzalez C, Noskov SY, Larsson HP. Molecular mechanism of Zn2+ inhibition of a voltage-gated proton channel. Proc Natl Acad Sci U S A. 2016;113:E5962-e5971.

8. Wu L, Wu G, Akhavan Sharif MR, Baker A, Jia Y, Fahey FH, Luo HR, Feener EP, Clapham DE. The voltage-gated proton channel Hv1 enhances brain damage from ischemic stroke. Nat Neurosci. 2012;15:565-73.

9. Tian DS, Li CY, Qin C, Murugan M, Wu LU, Liu JL. Deficiency in the voltagegated proton channel $\mathrm{Hv} 1$ increases M2 polarization of microglia and attenuates brain damage from photothrombotic ischemic stroke. J Neurochem. 2016;139:96-105.

10. Yu Y, Yu Z, Xie M, Wang W, Luo X. Hv1 proton channel facilitates production of ROS and pro-inflammatory cytokines in microglia and enhances oligodendrocyte progenitor cells damage from oxygen-glucose deprivation in vitro. Biochem Biophys Res Commun. 2018;498:1-8.

11. Weinlich R, Oberst A, Beere HM, Green DR. Necroptosis in development, inflammation and disease. Nat Rev Mol Cell Biol. 2017;18:127-36.

12. Fricker M, Tolkovsky AM, Borutaite V, Coleman M, Brown GC. Neuronal Cell Death. Physiol Rev. 2018;98:813-80.

13. Vande Walle L, Lamkanfi M. Pyroptosis. Curr Biol. 2016;26:R568-r572.

14. Zheng G, Zhan Y, Wang H, Luo Z, Zheng F, Zhou Y, Wu Y, Wang S, Wu Y, Xiang $G$, et al. Carbon monoxide releasing molecule-3 alleviates neuron death after spinal cord injury via inflammasome regulation. EBioMedicine. 2019:40:643-54

15. Yang J, Liu Z, Wang C, Yang R, Rathkey JK, Pinkard OW, Shi W, Chen Y, Dubyak GR, Abbott DW, Xiao TS. Mechanism of gasdermin D recognition by inflammatory caspases and their inhibition by a gasdermin D-derived peptide inhibitor. Proc Natl Acad Sci U S A. 2018;115:6792-7.

16. Coll RC, Holley CL, Schroder K. Mitochondrial DNA synthesis fuels NLRP3 inflammasome. Cell Res. 2018;28:1046-7.

17. Dolunay A, Senol SP, Temiz-Resitoglu M, Guden DS, Sari AN, Sahan-Firat S, Tunctan B. Inhibition of NLRP3 inflammasome prevents LPS-induced inflammatory hyperalgesia in mice: contribution of NF-kappaB, caspase-1/11, ASC, NOX, and NOS isoforms. Inflammation. 2017:40:366-86.

18. Yu Y, Luo X, Li C, Ding F, Wang M, Xie M, Yu Z, Ransom BR, Wang W. Microglial Hv1 proton channels promote white matter injuries after chronic hypoperfusion in mice. J Neurochem. 2020;152:350-67.

19. Guo Q, Li S, Liang Y, Zhang Y, Zhang J, Wen C, Lin S, Wang H, Su B. Effects of $\mathrm{C} 3$ deficiency on inflammation and regeneration following spinal cord injury in mice. Neurosci Lett. 2010;485:32-6.

20. Basso DM, Fisher LC, Anderson AJ, Jakeman LB, McTique DM, Popovich PG. Basso mouse scale for locomotion detects differences in recovery after spinal cord injury in five common mouse strains. J Neurotrauma. 2006;23: 635-59.

21. Anderson MA, O'Shea TM, Burda JE, Ao Y, Barlatey SL, Bernstein AM, Kim JH, James ND, Rogers A, Kato B, et al. Required growth facilitators propel axon regeneration across complete spinal cord injury. Nature. 2018:561:396-400.

22. Jing Y, Wu Q, Yuan X, Li B, Liu M, Zhang X, Liu S, Li H, Xiu R. Microvascular protective role of pericytes in melatonin-treated spinal cord injury in the C57BL/6 mice. Chin Med J (Engl). 2014;127:2808-13.

23. You L, Yang C, Du Y, Liu Y, Chen G, Sai N, Dong X, Yin X, Ni J. Matrine Exerts Hepatotoxic effects via the ROS-dependent mitochondrial apoptosis pathway and inhibition of Nrf2-mediated antioxidant response. Oxid Med Cell Longev. 2019;2019:1045345.

24. Ma P, Yan B, Zeng Q, Liu X, Wu Y, Jiao M, Liu C, Wu J, Yang X. Oral exposure of Kunming mice to diisononyl phthalate induces hepatic and renal tissue injury through the accumulation of ROS. Protective effect of melatonin. Food Chem Toxicol. 2014;68:247-56.

25. Gong Z, Pan J, Shen Q, Li M, Peng Y. Mitochondrial dysfunction induces NLRP3 inflammasome activation during cerebral ischemia/reperfusion injury. J Neuroinflammation. 2018;15:242.

26. Shi J, Zhao Y, Wang K, Shi X, Wang Y, Huang H, Zhuang Y, Cai T, Wang F, Shao F. Cleavage of GSDMD by inflammatory caspases determines pyroptotic cell death. Nature. 2015;526:660-5.

27. Jiang W, Li M, He F, Zhou S, Zhu L. Targeting the NLRP3 inflammasome to attenuate spinal cord injury in mice. J Neuroinflammation. 2017;14:207.

28. Chen X, Cui J, Zhai X, Zhang J, Gu Z, Zhi X, Weng W, Pan P, Cao L, Ji F, et al. Inhalation of hydrogen of different concentrations ameliorates spinal cord injury in mice by protecting spinal cord neurons from apoptosis, Oxidative injury and mitochondrial structure damages. Cell Physiol Biochem. 2018;47: $176-90$.

29. Nunomura A, Perry G, Pappolla MA, Wade R, Hirai K, Chiba S, Smith MA. RNA oxidation is a prominent feature of vulnerable neurons in Alzheimer's disease. J Neurosci. 1999;19:1959-64.

30. Zhou B, Zhang JY, Liu XS, Chen HZ, Ai YL, Cheng K, Sun RY, Zhou D, Han J, Wu Q. Tom20 senses iron-activated ROS signaling to promote melanoma cell pyroptosis. Cell Res. 2018;28:1171-85.

31. Frank D, Vince JE. Pyroptosis versus necroptosis: similarities, differences, and crosstalk. Cell Death Differ. 2019;26:99-114.

32. Cookson BT, Brennan MA. Pro-inflammatory programmed cell death. Trends Microbiol. 2001;9:113-4.

33. Liu W, Chen Y, Meng J, Wu M, Bi F, Chang C, Li H, Zhang L. Ablation of caspase-1 protects against TBI-induced pyroptosis in vitro and in vivo. J Neuroinflammation. 2018;15:48.

34. Ding J, Wang K, Liu W, She Y, Sun Q, Shi J, Sun H, Wang DC, Shao F. Poreforming activity and structural autoinhibition of the gasdermin family. Nature. 2016;535:111-6.

35. Kroner A, Greenhalgh AD, Zarruk JG, Passos Dos Santos R, Gaestel M, David S. TNF and increased intracellular iron alter macrophage polarization to a detrimental M1 phenotype in the injured spinal cord. Neuron. 2014;83:1098-116.

36. Askari VR, Shafiee-Nick R. The protective effects of beta-caryophyllene on LPS-induced primary microglia M1/M2 imbalance: a mechanistic evaluation. Life Sci. 2019;219:40-73.

37. Zhang L-M, Zhang D-X, Fu L, Li Y, Wang X-P, Qi M-M, Li C-C, Song P-P, Wang $X-D$, Kong $X$-J. Carbon monoxide-releasing molecule-3 protects against cortical pyroptosis induced by hemorrhagic shock and resuscitation via mitochondrial regulation. Free Radic Biol Med. 2019;141:299-309.

38. Diao C, Chen Z, Qiu T, Liu H, Yang Y, Liu X, Wu J, Wang L. Inhibition of PRMT5 attenuates oxidative stress-induced pyroptosis via activation of the Nrf2/HO-1 signal pathway in a mouse model of renal ischemia-reperfusion injury. Oxid Med Cell Longev. 2019;2019:2345658.

39. Miao EA, Rajan JV, Aderem A. Caspase-1-induced pyroptotic cell death. Immunol Rev. 2011;243:206-14.

40. Jiang W, Huang Y, Han N, He F, Li M, Bian Z, Liu J, Sun T, Zhu L. Quercetin suppresses NLRP3 inflammasome activation and attenuates histopathology in a rat model of spinal cord injury. Spinal Cord. 2016;54:592-6.

41. Zendedel A, Johann S, Mehrabi S, Joghataei MT, Hassanzadeh G, Kipp M, Beyer C. Activation and regulation of NLRP3 inflammasome by intrathecal application of SDF-1a in a spinal cord injury model. Mol Neurobiol. 2016;53: 3063-75.

42. Zhong Z, Liang S, Sanchez-Lopez E, He F, Shalapour S, Lin XJ, Wong J, Ding $\mathrm{S}$, Seki E, Schnabl B, et al. New mitochondrial DNA synthesis enables NLRP3 inflammasome activation. Nature. 2018:560:198-203.

43. Latz E. The inflammasomes: mechanisms of activation and function. Curr Opin Immunol. 2010;22:28-33.

44. Beattie MS, Farooqui AA, Bresnahan JC. Review of current evidence for apoptosis after spinal cord injury. J Neurotrauma. 2000;17:915-25.

45. McTigue DM, Tripathi RB. The life, death, and replacement of oligodendrocytes in the adult CNS. J Neurochem. 2008;107:1-19.

46. Bok E, Chung YC, Kim KS, Baik HH, Shin WH, Jin BK. Modulation of M1/M2 polarization by capsaicin contributes to the survival of dopaminergic neurons in the lipopolysaccharide-lesioned substantia nigra in vivo. Exp Mol Med. 2018;50:76.

\section{Publisher's Note}

Springer Nature remains neutral with regard to jurisdictional claims in published maps and institutional affiliations. 